\title{
XVIII. Ueber die Rationalität einer dreizähligen Symmetrieaxe.
}

\author{
Von \\ Vic. de Souza-Brandão in Lissabon.
}

Nachdem in dieser Zeitschr. 1\%, 617 ein Referat von Herrn G. Wulff uber einen strengen Beweis fur die Rationalität dreizähliger Symmetrieaxen, welcher von Herrn Fedorow in den Verhandlungen der russischen mineralogischen Gesellschaft veröffentlicht wurde, erschienen ist, wurde seitens des Herrn Hecht (Neues Jahrbuch für Mineralogie 1893, 2, 173) oine Berichtigung jenes Beweises und später wieder (Ebenda, 1894, 1, 199) von Fedorow eine Vertheidigung seines Beweises bekannt gemacht. Da keiner von diesen Gelehrten uns sagt, worin der Fehler seines Gegners liegt, so ist aus den erwähnten Aufsätzen nicht ohne Weiteres zu ersehen, welcher von Beiden Recht hat. Ich erlaube mir deshalb meine eigene Ansicht mitzutheilen, in der Hoffnung, Anderen vielleicht die Bemühungen zu ersparen, die ich selbst hatte.

Drei unter einander gleichgeneigte Kanten sind uberhaupt krystallographisch möglich. Betrachtet man sie als Fundamentalkanten, so werden dadurch nur drei Elemente - die Fundamentalwinkel - bestimmt, und es bleiben die Axenverhältnisse noch durch eine hinzuzuziehende Fläche oder Kante zu berechnen. Werden aber die drei unter einander gleichgeneigten Kanten, welche wir mit $x_{1}, x_{2}, x_{3}$ bezeichnen wollen, als mit Bezug auf eine dreizählige Axe $\alpha$ symmetrisch vorausgesetzl, d. h. sind $x_{2}$ und $x_{3}$ als aus $x_{1}$ durch Drehungen ron $120^{\circ}$ und $240^{\circ}$ um eine Gerade $\alpha$ erzeugt anzusehen, so fragt sich, ob dadurch der Complex vollständig bestimmt ist oder nicht, d. h. ob damit die Axenverhältnisse, ohne Benulzung irgend einer neuen Fläche oder Kante, keiner Unbestimmtheit mehr unterliegen.

Bei der Entscheidung dieser Frage haben die Krystallographen zwei Ricbtungen befolgt, die man vielleicht die physikalische und die rein mathematische nennen könnte. Der ersten Auffassung nach, welche 
in dem vortrefflichen Werke von Liebisch (Physikalische Krystallographie, 1891 , S. 11) und schon in seiner etwas alteren geometrischen Krystallographie (1881, S. 195, 196) vertreten ist, wird die Eigenschaft rationaler Axenverhätnisse als eine dem Grundgesetze der physikalischen Krystallographie unterworfene angesehen, woraus für unseren Fall folgt, dass, wenn zwei von drei unter einander gleichgeneigten Kanten durch die dritte und die Operationen einer dreizähligen Symmelrieaxe erzeugt worden sind, jede Ebene eine mögliche Fläche des von jenen drei Kanten bestimmten Complexes ist, welche auf letzteren Abschnilte bildet, deren Verhältnisse rational sind, und jede Richtung eine mögliche Kante, deren axoparallele Coordinaten mit Bezug auf die drei jene Kanten verbindenden Ebenen rationale Verhältnisse haben. Da nun für die dreizählige Axe alle drei Coordinaten gleich sind, ebenso wie die Kantenabschnitte für die zu ihr normale Ebene (die Basis), so ist obne Weiteres festgestellt, dass dreizählige Axe und dazu normale Ebene mögliche Kante und mögliche Flyche sind.

Diese Auffassung scheint mir die richtige zu sein, da die Gleichwerthigkeit von Richtungen und Ebenen, auf Grund der Erfahrung und physikalischer Betrachtung gefunden, nicht deshalb aufhört für eine besondere Eigenschaft (welche nichts anderes als eine physikalische sein kann) zu gelten, weil ein allgemeineres Grundgesetz - das sogenannte Grundgesetz der geometrischen Krystallograpbie - nicht eine solche Anforderung von vornherein an gleichwerthige Richtungen stellt. Wenn ein Symmelrieelement als ein solches, durch dessen Operation ein Complex in sich selbst ubergeht, definirt wird, so ist nicht begreiflich, wie man die Rationalität der Axenverbältnisse auf gleichwerthigen Kanten, identisch mit der Gleichheit der krystallographischen Einheiten dieser Kanten, von der Eigenschaft, jenes Symmetrieelement zu besitzen, trennen kann.

Aus dem Gesagten folgt auch sohon, dass, soll die dreizählige Axe eine mögliche Kante sein, die Axenverhältnisse, wenn als Fundamentalkanten drei gleichwerthige Kanten angenommen werden, rational sein mussen.

Die rein mathematische Richtung, von Fedor ow und $\mathrm{Hech}$ t verfolgt, verwirft fur die Entscheidung dieser Frage die Gultigkeit des Grundgesetzes der physikalischen Krystallographie und lässt nur das Gesetz der rationalen Indices gelten.

Fúr die Anwendung des letzteren ist die Hinzuziehung weiterer zwei Kanten nothwendig, welche natürlich mit der dreizähligen Axe nicht zusammenfallen durfen. Durch die Annahme einer dreizähligen Symmetrieaxe werden aber zu einer Kante $o$ noch zwei andere $q$ und $r$ bedingt, welche unter sich und mit $o$ einerseits, und alle drei mit der Axe andererseits gleiche Winkel bilden. Da aber die vier Kanten $x_{1}, x_{2}, x_{3}$, o den Complex vollständig bestimmen, so fragt sich, ob und in welchen Fällen $q$ und $r$ mögliche Kanten sind. 
Wir nehmen an, dass die Drehungen um die dreizählige Axe, welche $o$ in $q$ und $q$ in $r$ auf dem kleinsten Wege überführen, dem Sinne nach mit denjenigen ubereinstimmen, welche $x_{1}$ in $x_{2}$ und $x_{2}$ in $x_{3}$ ebenfalls auf dem kleinsten Wege zur Goincidenz bringen. Dann genügt es, die Möglichkeit von $r$ zu untersuchen, da die Winkel unter den fún Kanten $x_{2}, x_{3}$, $x_{1}, o, r$ entsprechend gleich sind denjenigen, welche von $x_{1}, x_{2}, x_{3}, r, q$ gebildet werden, und in Folge dessen, wenn die Indices von $r$ mit Bezug auf $x_{2}, x_{3}, x_{1}, o$ rational sind, auch diejenigen von $q$ mit Bezug auf $x_{1}, x_{2}, x_{3}, r$.

Ist nun in der That $r$ eine mögliche Kante, so ist der eine dreizählige Symmetrieaxe besitzende Complex rational; ist dagegen $r$ keine mögliche Kante, so ist der dreizählig axosymmetrische Complex kein rationaler, oder mit anderen Worten: der rationale Complex, welcher durch die vier Kanten $x_{1}, x_{2}, x_{3}, o$, von welchen die drei ersten unter einander gleichgeneigt sind, bestimmt ist, wird keine dreizählige Symmetrieaxe besitzen.

Um nach der Rationalitätsbedingung von $r$ zu suchen, benutzen Fe dorow und Hecht den Fundamentalsatz der goniometrischen Krystallographie, welcher in der Rationalităt des dreifachen Verhältnisses von funf Flächen oder Kanten eines Complexes besteht. Werden mit $r_{1}, r_{2}, r_{3}$ die Indices von $r$, bezogen auf $x_{1}, x_{2}, x_{3}$ als Fundamentalkanten und $o$ als Einheitskante, und mit $X_{1}, X_{2}, X_{3}$ die Normalen der Axenebenen $x_{2} x_{3}, x_{3} x_{1}$, $x_{1} x_{2}$ bezeichnet, so gelangt $F$ edorow zu der Formel :

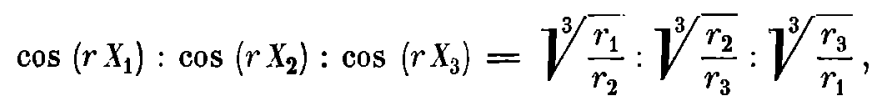

woraus er schliesst, dass die dreizählige Axe eine mögliche Kante ist, da die Gleichung für $\left(r X_{1}\right)=\left(r X_{2}\right)=\left(r X_{3}\right)$, die Form:

$$
\sqrt[3]{\frac{r_{1}}{r_{2}}}: \sqrt[3]{\frac{r_{2}}{r_{3}}}: \sqrt[3]{\frac{r_{3}}{r_{1}}}=1: 1: 1
$$

- annehmend, durch rationale Werthe der Verhältnisse $\frac{r_{1}}{r_{2}}, \frac{r_{2}}{r_{3}}, \frac{r_{3}}{r_{1}}$ befriedigt wird.

Hecht wendet ein, dass der vorhergehende Beweis, zur Hälfte gefuhrt, nichts beweise, und den erwähnten Fundamentalsatz auf die Kanten $x_{1}, x_{2}, x_{3}, r$ und $o^{\prime}$ (zunächst beliebig) weiter anwendend, kommt er schliesslich zu den Gleichungen:

$$
\begin{aligned}
& \cos \left(o^{\prime} X_{1}\right): \cos \left(o^{\prime} X_{2}\right): \cos \left(o^{\prime} X_{3}\right)= \\
& r_{1}{ }^{\prime} \sqrt[3]{\frac{r_{1}}{r_{2}}}: r_{2}{ }^{\prime} \sqrt[3]{\frac{r_{2}}{r_{3}}}: r_{3}{ }^{\prime} \sqrt[3]{\frac{r_{3}}{r_{1}}},
\end{aligned}
$$

wo $r_{1}{ }^{\prime}, r_{2}{ }^{\prime}, r_{3}{ }^{\prime}$ ganze rationale Zahlen sind. Indem er nun die beliebige Kante $o^{\prime}$ mit der dreizähligen Axe identificirt, findel er: 


$$
\left(r_{1}^{\prime}\right)^{3}:\left(r_{2}^{\prime}\right)^{3}:\left(r_{3}^{\prime}\right)^{3}=\frac{r_{2}}{r_{1}}: \frac{r_{3}}{r_{2}}: \frac{r_{1}}{r_{3}}
$$

d. h. es müssen die Verhältnisse der Indices der Kante $r$, von einem Proportionalitätsfactor abgesehen, gleich den dritten Potenzen dreier ganzer rationaler Zahlen sein.

Hech t betrachtet die Gleichungen (1) für sich allein, als die Bedingung dafür, dass ein Complex eine dreizahlige Symmetrieaxe besitze, was (1) auch jedenfalls beweist; als Beweis fur die Zugehörigkeit der Axe zum Complex aber sieht er nur Formel (2) an. Mir scheint es auch, dass (1), der Bedeutung von $r_{1}, r_{2}, r_{3}$ wegen, nur bezuglich einer mit der Einheitskante gegen die dreizählige Axe symmetrischen Kante Gultigkeit behält, uber die Abhängigkeit zu verschiedenen einfachen Formen gehörender Kanten von einander jedoch nichts aussagt. Jedenfalls ist nichl gestattet, (1) auf die Axe selbst anzuwenden, da dann $r$ und o (Einheitskante) coincidiren werden, und es natürlich nichts Merkwürdiges hat, dass man von einer als möglich angenommenen Kante $o$ findet, dass sie, wenn auch mit einer anderen Bezeichnung $(r)$, möglich ist. Der Satz von Fedorow beweist also nicht genug. Wir werden sehen, dass derjenige von $\mathrm{Hecht}$ zu viel beweist. Ich möchte an diesem Platze aber schon erwähnen, dass F ed orow zu einem Resultate gelangt ist, welches nach gegenwärtiger Untersuchung richtig ist, trotzdem es, wie oben gezeigt, nicht als eine zwingende Folge seiner Erörterungen betrachtet werden kann, nämlich, dass für e inen bestimmten Winkel $\left(x_{2} x_{3}\right)=\left(x_{3} x_{1}\right)=\left(x_{1} x_{2}\right)$ ein und nur ein rationaler Gomplex existirt, und dass diesemeinzigen rationalen Complex auch die dreizählige Axe als Kante angehört. Dem weiter unten zu discutirenden Beweise von $\mathrm{Hech}$ t nach soll es aber unendlich viele rationale Complexe geben und darunter nur einen, welcher die dreizählige Axe als Kante enthält.

Wir wollen nun auf analytischem Wege einen für unseren Gegenstand wichtigen uud sogar entscheidenden Satz ableiten, den wir oben physikalisch begrúndet haben. Aus den Verhältnissen (Liebisch, Geometr. Krystallogr, S. 75)

$$
\begin{gathered}
a_{1} \eta_{1}: a_{2} \eta_{2}: a_{3} \eta_{3} \\
=\sin \left(\pi_{2} \pi_{3}\right) \cos \left(\eta p_{1}\right): \sin \left(\pi_{3} \pi_{1}\right) \cos \left(\eta p_{2}\right): \sin \left(\pi_{1} \pi_{2}\right) \cos \left(\eta p_{3}\right),
\end{gathered}
$$

worin $a_{1}, a_{2}, a_{3}$ die Axeneinheiten, $\eta_{1}, \eta_{2}, \eta_{3}$ die Indices einer beliebigen Kante $\eta, \pi_{1}, \pi_{2}, \pi_{3}$ die Fundamentalkantenrichtungen und $p_{1}, p_{2}, p_{3}$ die Normalenrichtungen der Axenebenen bedeuten, folgt fur die dreizählige Axe, für welche

$$
\cos \left(\eta p_{1}\right): \cos \left(\eta p_{2}\right): \cos \left(\eta p_{3}\right)=1: 1: 1,
$$

wenn man zu Fundamentalkanten die unter einander gleichgeneigten Kanten $x_{1}, x_{2}, x_{3}$ nimmt : 
woraus :

$$
a_{1} \eta_{1}: a_{2} \eta_{2}: a_{3} \eta_{3}=1: 1: 1
$$

$$
\eta_{i}=\frac{1}{a_{i}} \quad[i=1,2,3, \ldots]
$$

d. h. die dreizählige Axe ist rational und nur dann, wenn die Axenverhältnisse es auch sind. Und da dann jede durch rationale Axenschnittsverhältnisse charakterisirte Ebene oder durch rationale Coordinatenverbältnisse charakterisirte Richtung eine mögliche Fläche resp. Kante ist, so folgt hieraus, dass für einen bestimmten Winkel $\left(x_{n} x_{n+1}\right)[n=1,2,3, \ldots]$ der gleichwerthigen Fundamentalkanten es einen und nur einen rationalen Gomplex giebt, welcher zugleich die dreizählige Axe enthäl; er wird durch rationale Verhältnisseder Axenabschnitte jeder seiner Flächen und der axoparallelen Coordinaten jeder seiner Kanten gekennzeichnet.

Um unsere obige Behauptung vollständig zu begründen, bleibt noch übrig zu beweisen, dass, soll ein Complex utberhaupt dreizäblig axosymmetrisch sein, auch diese Bedingung der Rationalität der Axenabschnittsund Coordinatenverháltnisse erfüllt sein muss, d. h. dass die, sonst möglicherweise verschiedenen, rationalen Complexe mit einander und mit jenem identisch sind. Dies werden wir bei der nun folgenden Kritik des Hechtschen Beweises zeigen. Aus (1) und (3) folgt:

und da :

$$
\cos \left(r X_{1}\right): \cos \left(r X_{2}\right): \cos \left(r X_{3}\right)=\frac{1}{r_{1}^{\prime}}: \frac{1}{r_{2}^{\prime}}: \frac{1}{r_{3}^{\prime}} ;
$$

$$
\left(r X_{1}\right)=\left(o X_{2}\right), \quad\left(r X_{2}\right)=\left(o X_{3}\right), \quad\left(r X_{3}\right)=\left(o X_{1}\right),
$$

so erhält man, indem die linke Seite mit $\sin \left(x_{3} x_{1}\right)=\sin \left(x_{1} x_{2}\right)=$ $\sin \left(x_{2} x_{3}\right)$ multiplicirt wird:

$$
\begin{array}{r}
\sin \left(\dot{x}_{3} x_{1}\right) \cos \left(o X_{2}\right): \sin \left(x_{1} x_{2}\right) \cos \left(o X_{3}\right): \sin \left(x_{2} x_{3}\right) \cos \left(o X_{1}\right) \\
=\frac{1}{r_{1}^{\prime}}: \frac{1}{r_{2}{ }^{\prime}}: \frac{1}{r_{3}{ }^{\prime}} .
\end{array}
$$

Die Ausdrucke links sind nichts Anderes, als die homogenen Coordinaten der Kante 0 , bezogen auf die Fundamenlalkanten $x_{2}, x_{3}, x_{1}$ und die Einbeitskante o selbst, also mit dem Symbol [111]. Wir müssen desbalb) in dem Ausdrucke der homogenen Coordinaten (Liebisch, Geometr. Krystallogr. S. 74) setzen :

so dass :

und schliesslich :

$$
\eta_{1}=\eta_{2}=\eta_{3}
$$

$$
\xi_{1}: \xi_{2}: \xi_{3}=a_{1}: a_{2}: a_{3}
$$

$$
a_{1}: a_{2}: a_{3}=\frac{1}{r_{1}^{\prime}}: \frac{1}{r_{2}^{\prime}}: \frac{1}{r_{3}^{\prime}} .
$$

Die Gleichung (3) fübrt also direct zu dem Schlusse, dass, damit die dreizählige Axe rational sei, die Axenverhältnisse des Complexes rational sein müssen, welches auch die Einheitskante sein möge, was mit unserem 
obigen Satze ubereinstimmt; und insofern ist der Beweis von Hecht richtig. Dieser Forscher behauptet aber, dass die von Fedorow aufgestellte Gleichung (1) nicht dasselbe beweise, wie (3), sondern einfach die Bedingung furr die Rationalität eines durch die Kanten $x_{1}, x_{2}, x_{3}$, o bestimmten Complexes darstelle, deren es mehrere giebt, die im Allgemeinen aber die dreizählige Axe nicht enthalten.

Wir wollen nun zeigen, dass diese Behauptung falsch ist, und dass (1) auch weiter nichts bedeutet, als die Rationalität jener Axenverhältuisse, also die Goincidenz aller rationalen Complexe in einem einzigen, welchem, wie mehrfach gezeigt, die dreizählige Axe angehört. In der That sind, wenn mit $a_{1}: a_{2}: a_{3}$ die von der Kante $o$ als Einheitskante auf $x_{1}, x_{2}, x_{3}$ bestimmten Axenverhältnisse bezeichnet worden, resp. durch

$$
a_{1}, a_{2}, a_{3} \text { und } a_{1} r_{1}, a_{2} r_{2}, a_{3} r_{3}
$$

die homogenen Coordinaten von $o[111]$ und $r\left[r_{1} r_{2} r_{3}\right]$ auszudrucken. Der Stellung von $o$ und $r$ gegen $x_{1}, x_{2}, x_{3}$ halber ist aber:

$$
a_{1} r_{1}=a_{2}, \quad a_{2} r_{2}=a_{3}, \quad a_{3} r_{3}=a_{1} \quad \text { und } \quad r_{1} r_{2} r_{3}=1 \text {. }
$$

Hieraus folgt weiter :

und :

$$
\frac{r_{1}}{r_{2}}=\frac{\left(a_{2}\right)^{2}}{a_{3} a_{1}}, \quad \frac{r_{2}}{r_{3}}=\frac{\left(a_{3}\right)^{2}}{a_{1} a_{2}}, \quad \frac{r_{3}}{r_{1}}=\frac{\left(a_{1}\right)^{2}}{a_{2} a_{3}}
$$

oder

$$
\left(a_{1}\right)^{3}:\left(a_{2}\right)^{3}:\left(a_{3}\right)^{3}=\frac{r_{3}}{r_{1}}: \frac{r_{1}}{r_{2}}: \frac{r_{2}}{r_{3}}
$$

$$
a_{1}: a_{2}: a_{3}=\sqrt[3]{\frac{r_{3}}{r_{1}}}: \sqrt[3]{\frac{r_{1}}{r_{2}}}: \sqrt[3]{\frac{r_{2}}{r_{3}}} .
$$

Multiplicirt man die rechte Seite mit :

$$
r_{2} \sqrt[3]{\left(r_{3}\right)^{2} r_{1}}=\sqrt[3]{\frac{r_{2}}{r_{1}}}=\sqrt[3]{\left(r_{2}\right)^{2} r_{3}},
$$

was leicht aus (4) hervorgeht, so erhält man schliesslich

$$
a_{1}: a_{2}: a_{3}=r_{2} r_{3}: 1: r_{2} \text {, }
$$

ohne Benutzung der Hecht'schen Zahlen $r_{1}{ }^{\prime}, r_{2}{ }^{\prime}, r_{3}{ }^{\prime}$.

Dies lässt sich auch direct aus (4) ableiten, da:

und schliesslich:

$$
\frac{a_{2}}{a_{3}}=\frac{1}{r_{2}}, \quad \frac{a_{1}}{a_{3}}=\frac{r_{3}}{1}=\frac{r_{2} r_{3}}{r_{2}}
$$

$$
a_{1}: a_{2}: a_{3}=r_{2} r_{3}: 1: r_{2} \text {. }
$$

Damit ist der zweite wichtige Sat $z$ bewiesen; welcher mit dem ersten unsere obige Behauptung ausmacht.

Der Satz von Fedorow ist richtig; Letzterer ist aber nicht weit genug 
mit seinem Beweise gegangen, und da er nicht nach den Axeneinheiten suchte, so hat er nichts bewiesen. Dagegen ist Hecht, ohne sich bei denselben aufzuhalten, zu weit gegangen, und konnte deshalb nicht die wahre Lösung finden.

Das trigonale System ist also in dieser Beziehung, wie in allen, welche von der Geradzähligkeit der Hauptaxe unabhängig sind, den anderen durch eine Hauplaxe ausgezeichneten Systemen analog, d. h. es werden durch zwei nach der Hauptaxe gleichwertbige Elemente - Flächen oder Kanten die geometrischen Constanten vollständig beslimmt, und unter den möglichen Kanten und Flächen befinden sich stets die Hauptaxe und die zu ihr normale Ebene.

Erst nach Uebergabe obigen Aufsatzes an die Redaction dieser Zeitschrift hatte ich das Vergnügen, eine Abhandlung von Herrn Fedorow: Das Grundgesetz der Krystallograpbie (diese Zeitschr. 23, 99), zu lesen, welche, trotzdem sie uns nichts Neues bezuglich des Beweises der Rationalităt trigonaler Axen auf Grund des geometrischen Gesetzes bringt, mich bewogen hat, einige Bemerkungen uber die darin angeführten Ideen meinem Aufsatze hinzuzufugen.

Herr F edorow stellt die Structurtheorien oder, wenn man will, ihren grundlegenden Gedanken an die Spitze unserer Wissenschaft und leitet daraus die beiden Hauptgesetze der Krystallographie - das Zonen- und Symmetriegesetz - deductiv ab. Jener grundlegende Gedanke der Structurtheorien - die regelmässige Vertheilung der Massenpunkte eines krystallisirten Körpers - wird von F edorow als das Grundgesetz der physikalischen Krystallographie angesehen und schliesslich zum Hauptgeselze der Krystallographie uberhaupt erhoben.

Dies dürfte aber nicht berechtigt sein. Das Zonen- oder Rationalitätsgesetz ist ein durch die Forschung vollkommen bestätigtes Erfahrungsgesetz, und wenn Fedorow glaubt, besonders wegen der Unsicberheit höherer indicirter Flächen, dass jemand daran zweifeln dürfte, so wäre die atomislische Theorie in der Chemie, mit der weit grösseren Unsicherheit der Verbindungsgewichte der Elemente, als eine auf falsche Erfahrung basirte Theorie zu bezeichnen. Diese Analogie ist aber nicht ganz treffend und die weitere Vergleichung nicht zu Gunsten der Structurtheorien. Die atomistische Vorstellung, auf jenen Erfahrungsgeselzen begrúndet, hat sich bald durch die einfache Erklärung mancher Thatsachen zu einer Theorie ausgebildet, welche, sich dann durch successive Aenderungen den hinzukommenden Erfahrungen anpassend, auf die weitere Forschung leitend wirkte und noch fortwirkt. Aus dem längst ausser Zweifel gesetzten Rationalitätsgesetze und der Gleichheit des physikalischen Verhaltens nach parallelen Richtungen im Krystalle ist inductiv die den Structurtheorien 
zu Grunde liegende Vorslellung der regelmässigen Massenpunktvertheilung entstanden, welche aber bis zur Gegenwart das Stadium einer Hypothese nicht uberschritten hat.

Das Rationalitätsgesetz war der Entstehung der sogenannten Structurtheorien nicht fremd, wie man vielleicht im Sinne der in Rede stehenden Abhandlung glauben möchte, da die den beiden consequent durchgeführten - der Brava is-Mallard'schen und der Sohncke-Schönflies'schen - Theorien vorangehende und dieselben fördernde $\mathrm{H}$ au y'sche Decrescenztheorie wesentlich darauf gegründet war, ohne besondere Rücksicht auf andere physikalische Eigenschaften. Wäre es aber nicht so, und wäre das Rationalitätsgesetz als eine deductive Prüfung der jenen Theorien zu Grunde liegenden Vorstellung zu betrachten, so wurde man doch nicht zugeben können, dass diesem Gesetze sein ursprünglicher Charakter ein es Erfahrungserwerbnisses entzogen werde.

Ich kenne sonst keine einzige, allgemein anerkannte, unbestreitbare, deductive Prifung irgend einer der beiden Structurtheorien und betrachte diese als nichts Materielles darbietende, rein mathematische Schöpfungen, welche dem Geiste ihrer Erfinder und Mitarbeiter die grösste Ehre machen, denen aber, wenigstens für die nächste Zeit, die krystallographische Forschung wenig Hilfe (was von einer Theorie in erster Linie zu fordern ist) zu verdanken haben wird. Auch Fedorow ist der Meinung, dass diese sogenannten Theorien einen solchen Namen nicht verdienen, wie man sich beim Lesen seiner werthvollen Abhandlung in dieser Zeitschr. 21, $287 \mathrm{ff}$. uberzeugen kann.

Beztiglich der verschiedenen Arten der Fassung des allgemeinen geometrischen Gesetzes möchte ich noch erwähnen, dass der Ausdruck durch die Doppelverhältnisse nicht mit dem sogenannten Rationalitäts- und Zonengesetze gleichbedeutend ist, wie schon daraus hervorgeht, dass ein sich auf die Elemente eines geometrischen Gebildes erster Art - des Ebenenbuischels resp. des ebenen Strahlenbüschels - beziehender Satz nicht mit einem anderen gleich extensiv und allgemein sein kans, welcher sich auf ein Gebilde zweiter Art, wie es das Bündel der Flächen und Kanten des Complexes ist, bezieht. Dem arithmetischen und dem geometrischen Ausdruck des geometrischen Grundgesetzes ist die goniometrische Fassung der Rationalität des dreifachen Verhältnisses von funf Krystallflächen oder Kanten, wovon das Gesetz des Doppelverhältnisses einen speciellen Fall bildet, an die Seite zu stellen. Dem goniometrischen Gesetze nach verhalten sich die drei Verhältnisse der Sinus der Ecken, welche zwei Flächen (Kanten) mit je zwei von drei anderen, unter sich nicht tautozonalen Flächen bilden, wie drei rationale ganze Zahlen $(H$ ech $t$, Neues Jahrb. f. Min. etc. 1888, 1, 75).

Nun gehe ich zum Symmetriegesetze uber. Wenn ich die Abhandlung 
von $\mathrm{Fed} \mathrm{orow} \mathrm{gul} \mathrm{interpretirt} \mathrm{habe,} \mathrm{so} \mathrm{ist} \mathrm{sein} \mathrm{Gedankengang} \mathrm{folgender:}$ Zunächst wird für die innere Structur der Krystalle die Hypothese der regelmässigen Vertheilung der Massenpunkte aufgestellt, darauf die möglichen Symmetriearten eines solchen Gitters oder Haufens untersucht, und da sie jene Symmetriearten in der That aufweisen können, so sei dadurch erst streng bewiesen, dass solche Symmetriearten für Krystalle wirklich möglich sind. Mir scheint, es wird hier aus einer reinen Hypothese, welche noch nicht einmal eine Theorie geliefert hat, wieder ein Gesetz deductiv abgeleitet und deshalb als strenger bewiesen angesehen, als durch die allein auf die Erfahrung begrundete, von irgend welcher Hypothese freie geometrische Betrachtung.

Nach dem Gesetze der Symmetrie zerfallen die Krystalle in verschiedene Gruppen, welche durch die Anzahl und Vertheilung der verschiedenen Richtungen, nach welchen die physikalischen Eigenschaften qualitativ und quantitativ gleich sind, charakterisirt werden. Unter diesen Eigenschaften ist diejenige der Krystallform als Ausdruck der Gesetzmässigkeit des Wachsthums die wenigst symmetrische und deshalb für die Natur des betreffenden Körpers die bezeichnendste; woraus hervorgeht, dass auch das physikalische Gesetz der Symmetrie nach den an der Krystallform gemachten Erfahrungen formulirt werden muss und formulirt worden ist. Das Hauptkriterium des Symmetriegesetzes ist bekanntlich die einfache Form, welche trotz aller der geselzmässigen Aeusserung dieses Kennzeichens entgegenwirkenden Nebenumständen doch zur Aufstellung der krystallographischen Symmetrielehre am meisten beigetragen hat.

Nach Fedorow's Meinung sei auch die Hypothese der regelmässigen Massenpunkt-Vertheilung nothwendig und ausreichend, nicht nur um zu beweisen, dass andere als die 32 bekannten Symmetriegruppen unmöglich sind, sondern auch, dass diese in der That möglich sind, weil sie mit obiger Hypothese vereinbar sind. Die geometrische Untersuchung der rationalen Krystallcomplexe hatte gezeigt, dass andere als jene 32 Gruppen unmöglich, diese also implicite möglich sind, indem sie mit dem die Erscheinung der einzelnen Flăche erfahrungsmässig allein beherrschenden Gesetze in Einklang stehen. Dies scheint mir mehr zu beweisen, als die Uebereinstimmung mit oder die Ableitung aus einer, wenn auch berechtigten Hypothese.

Die Structurtheorien können unmöglich das Symmetriegesetz in seiner Allseitigkeit in sich enthalten. Wählt man unter anderen Eigenschaften die Wachsthumserscheinungen, so sagt uns das erfahrungsmüssige Symmetriegesetz in seiner Fassung als Grenzgesetz, welche eine lang studirte und woblberechtigte Abstraction aus den thatsächlichen Verhältnissen darstellt, dass zum Vorhandensein einer Fläche das Vorhandensein anderer (der einfachen Form) nicht nur möglich, sondern nothwendig ist. Die genannten 
Theorien erfordern die Möglichkeit, bedingen aber nicht die actuelle Verwirklichung des Phänomens.

Das geometrische Gesetz ist gewiss auch ein physikalisches, da die Krystallform keine andere als eine physikalische Eigenschaft sein kann. Es wird abel unter jenem Namen ausgeschieden als sich nur auf eine Eigenschaft beziehend, welche durch Abstraction als eine geometrische betrachtet werden kann. Gewiss sind beide Gesetze, das geometrische wie das physikalische, als welches wir das Symmetriegesetz bezeichnen, partielle Ausdrücke des inneren Wesens des krystallisirten Stoffes, können daher zur deductiven Prufung für Hypothesen und Theorien dienen und diesen grosse Wahrscheinlichkeit verleihen, aber auf keinen Fall von solchen abgeleitet werden.

Um der Forschung eine sichere Grundlage zu liefern, muss man den Naturgesetzen ihren experimentellen Gharakter lassen. Alsdann können auf dieselben immer neue fruchttragendere Hypothesen aufgebaut werden, die in unserem Falle vielleicht besser als die actuellen Structurvorstellungen zur Entwickelung der wissenschaftlichen Krystallographie beitragen werden.

Es ist sehr bedenklich, wenn in elementaren Lehrbuchern, welche besonders den Anfängern gewidmet sind, Beweise von thatsächlichen Verhältnissen, von Erfahrungsgesetzen, wie z. B. das Zonengesetz, die auch mittelst einer wohlausgebildeten Theorie verwerflich wảren, auf Grund jener Structurvorstellungen gegeben werden. Solche Lehrbücher, welche von diesen Vorstellungen als unanfechtbaren Errungenschaften ausgehen und darauf die Darstellung der Krystallographie basiren, sind recht unzweckmässig; ich sehe als mustergiltige Methode diejenige an, die Prof. Liebisch in seinen zwei bekannten Werken der Krystallographie mit voller Strenge befolgt hat, und möchte, von veralteten Stellen abgesehen, diese Lehrbücher Allen empfehlen, welche ein gesundes Studium durchøuführen suchen. 